\title{
Editorial: Transitions Through Education
}

\author{
Elizabeth Fraser Selkirk Hannah * and Divya Jindal-Snape \\ School of Education and Social Work, University of Dundee, Dundee, United Kingdom
}

Keywords: education, children, young adults, young people, transitions

\section{Editorial on the Research Topic}

\section{Transitions Through Education}

Throughout our life span we go through a range of different types of transitions. These transitions can be construed from a positive and/or negative discourse, offering challenges and opportunities to grow and develop as human beings. This research topic focuses on the educational transitions of children, young people and young adults in compulsory and post-compulsory education. The aim was to identify and address gaps in the literature; offer new insights in the area; and provide recommendations for policy, practice and future research. In total, there are nine articles, comprising one literature review and eight empirical studies.

Jindal-Snape et al. identify a gap in previous literature reviews of primary-secondary transitions, namely none have focused on researchers' worldviews, use of theories/models and adoption of frameworks. The authors argue that it is important to understand researchers' conceptual frameworks and how these underpin their methodology, interpretation of findings and conclusions. Using a systematic mapping review process, the authors found that most researchers in the 96 papers did not offer their conceptualisation of transition and, of those that did, few used that conceptualisation to underpin the research design and interpretation of findings. The findings from this review have implications for developing a more robust research base which builds on and critiques researchers' theoretical frameworks in the field of educational transitions research.

Three articles focus on transitions during the compulsory education period. Two focus on primary to secondary school transition (Barlow; Stack et al.). Barlow explores the use of drama to support the development of positive peer relationships during this significant period of transition with associated changes in the educational curriculum, physical environment and social relationships with adults and peers. The author identifies a paucity of literature using drama pedagogy during primary-secondary transitions. Using a qualitative research paradigm, adopting Multiple and Multidimensional Transitions theory (MMT, Jindal-Snape, 2016), and gathering data before and after the move to secondary school in the Scottish context, the author found that the creative medium enabled pupils to explore their hopes and fears in a safe environment and resulted in the creation of friendships and reduction in anxieties associated with the move. The author recommends further research in the use of drama to support this transition, the adoption of longitudinal research designs, and designs seeking the perspectives of a range of stakeholders. Stack et al. investigate the experiences of children with Autism Spectrum Disorder during primary-secondary transition in Ireland. Drawing on Stage-Environment Fit theory (Eccles et al., 1993), and adopting a qualitative research paradigm, they sought the views of children and parents using semi-structured interviews. They find that the children were, on the whole, more positive about secondary school than primary school and recommend that transition support should be individualised to the needs of the child rather than adopting a universal approach. The third paper (Dunlop), drawing on quantitative and qualitative data from a longitudinal study in one Scottish local authority, 
explores the relationship between wellbeing, attainment, and transitions experiences from pre-school to leaving school and outcomes for young people leaving school. The complexity and individual nature of transitions experiences are highlighted. The author concludes that the concept of "wellbeing needs" should be better defined and adopted and that the concept of attainment has tended to be overly restricted to literacy and maths which may neglect areas where children are more successful.

Four papers focus on the transitions of students in postcompulsory education. Willems et al., in a study conducted in Belgium, focus on the transition of students into the first year of university courses leading to a professional qualification, an area under-represented in extant literature. The authors offer a critical perspective on conceptualisations and the theoretical basis for transitions research in this area and aim to add to our understanding of the processes underpinning students' experiences utilising a qualitative methodology. The authors found a complex inter-relationship between the themes and sub-themes which emerged in the findings and, similar to Stack et al.; Dunlop, commented on the individualised nature of the experiences. Bethel et al. focus on the transitions of international students entering higher education in New Zealand. The authors highlight the multiple transitions (e.g., cultural, educational, social, practical) experienced by students and the potential impact on psychological well-being. The authors are interested in the role of contextual factors which are open to change, such as connectedness, and focus on the mediational role of host national connectedness on the psychological adaptation of international students. Using path analysis to test a predictive model, the authors conclude that host national connectedness is a "complex, multi-faceted construct." Glazzard et al. investigated the transitions of five individuals who identify as lesbian, gay, bisexual, trans, or queer (LGBTQ+) as they enter and progress through higher education. Conceptualising transitions using MMT theory ((Jindal-Snape, 2016), adopting a longitudinal research design, and using a range of methods, the authors found that individuals' transition experiences were largely positive in contrast with the "tragic narratives" in extant

\section{REFERENCES}

Eccles, J. S., Midgley, C., Wigfield, A., Buchanan, C. M., Reuman, D., Flanagan, C., et al. (1993). Development During Adolescence: The Impact of StageEnvironment Fit in Young Adolescents' Experiences in Schools and Families. Am. Physol. 48, 90-101. doi:10.1037//0003-066x.48.2.90

Jindal-Snape, D. (2016). A-Z of Transitions. London, UK: Palgrave Macmillan.

Conflict of Interest: The authors declare that the research was conducted in the absence of any commercial or financial relationships that could be construed as a potential conflict of interest. literature. The article highlights the multiple and multidimensional transitions of the students over a 3-year period and the significance of identity. The authors recommend a cross-university approach to curriculum design and importance of institutional ethos to support LGBTQ+ students. Mateu et al. offer an insight into the transitions of elite athletes moving into higher education, focusing on a specific degree course, namely Physical Activity, and Sport Sciences (PASS). The researchers set out to investigate participants' perceptions of the negative (barriers and demands) and positive (resources) aspects of the experience, as well as the differential transition pathways. The authors recommend that educational institutions and sports organisations enhance existing approaches to help elite athletes navigate a degree course with both practical and educational components.

Finally, one paper looks at the transitions of early career academics (ECAs) in higher education (Rientes and Hosein). The authors use Social Network Analysis to investigate the lived experiences of individuals who participated in a professional development programme. They found that ECAs tended to find support from their partners or individuals at the same hierarchical level rather than individuals in senior management roles. The authors propose that institutions should consider the development of additional spaces to support the professional development of ECAs and recommend that future research should focus on the social networks of mid-career academics.

To conclude, despite research being conducted in different countries and the adoption of a range of research paradigms, methodologies and methods, authors have highlighted the multiple nature of transitions; offered a balanced discourse; and recommended the importance of individual transitions and individualised support.

\section{AUTHOR CONTRIBUTIONS}

All authors listed have made a substantial, direct, and intellectual contribution to the work and approved it for publication.

Publisher's Note: All claims expressed in this article are solely those of the authors and do not necessarily represent those of their affiliated organizations, or those of the publisher, the editors and the reviewers. Any product that may be evaluated in this article, or claim that may be made by its manufacturer, is not guaranteed or endorsed by the publisher.

Copyright (c) 2022 Hannah and Jindal-Snape. This is an open-access article distributed under the terms of the Creative Commons Attribution License (CC BY). The use, distribution or reproduction in other forums is permitted, provided the original author(s) and the copyright owner(s) are credited and that the original publication in this journal is cited, in accordance with accepted academic practice. No use, distribution or reproduction is permitted which does not comply with these terms. 